\title{
Pisot and Salem Numbers in Intervals of the Real Line*
}

\author{
By David W. Boyd
}

\begin{abstract}
Based on the work of Dufresnoy and Pisot, we develop an algorithm for determining all the Pisot numbers in an interval of the real line, provided this number is finite. We apply the algorithm to the problem of determining small Salem numbers by Salem's construction, and to the proof that certain Pisot sequences satisfy no linear recurrence relation.
\end{abstract}

Introduction. A real algebraic integer $\theta>1$ is a Pisot (or Pisot-Vijayaraghavan) number if all its other conjugates lie strictly within the unit circle. The set of such numbers, $S$, is known to be closed in the real line [12], and has a known minimum strictly greater than 1 [14]. In [7], Dufresnoy and Pisot introduced a powerful method for investigating $S$ and used it to determine all numbers in $S \cap[1, \tau+\epsilon]$, where $\tau=\left(1+5^{1 / 2}\right) / 2$ and $0<\epsilon<0.0004$. They showed that $\tau$ is the smallest limit point of $S$ and is an isolated point of $S^{\prime}$. Subsequently, their method has been used to investigate the successive derived sets of $S[1],[5],[8],[10]$.

The set $T$ of Salem numbers consists of those algebraic integers $\sigma>1$ for which all other conjugates lie within or on the unit circle, with at least one conjugate on the circle. As a consequence, $\sigma$ satisfies a reciprocal equation with all but two of its roots (namely $\sigma$ and $\sigma^{-1}$ ) on the unit circle [13]. In contrast to $S$, very little is known about $T$, although it has been conjectured that inf $T>1$ and even that $S \cup T$ is a closed set [3], [4].

By a construction due to Salem [13], given $\theta \in S$, two infinite sequences $\theta_{m}^{+}$and $\theta_{m}^{-}$of points of $T$ are produced. We showed in [4] that this construction in fact gives all members of $T$. Furthermore, if $m \geqslant 2$ then $\theta_{m}^{ \pm} \rightarrow \infty$ as $\theta \rightarrow \infty$. In this paper, we shall show how the methods of [7] can give more precise results of this type.

As a byproduct of the various computations described in this paper, we have discovered four new Salem numbers in the interval $[1,1.3]$ to add to the list of 39 given in [4].

A related motivation for this paper comes from the study of Pisot sequences ( $E$ sequences). The Pisot sequence $E\left(a_{0}, a_{1}\right)$ is the sequence of positive integers defined by $a_{n}=N\left(a_{n-1}^{2} / a_{n-2}\right)(n \geqslant 2)$, where $N(x)=[x+1 / 2]$ is the function which rounds $x$ to the "nearest" integer. These sequences are intimately related to the sets $S$ and $T$ since if $\left\{a_{n}\right\}$ satisfies a linear recurrence relation then the defining equation has a member of $S$ or $T$ as its only root in $|z|>1$. We showed in [3] that there are many $E$-sequences which are nonrecurrent. However, specific examples were difficult to give

Received September 7, 1977.

AMS (MOS) subject classifications (1970). Primary 12A15; Secondary 12A04, 10 F40.

* This research was supported in part by a grant from the Canadian N.R.C. and an I. W. Killam Senior Research Fellowship. 
because of a lack of detailed knowledge of $S$ for $\theta>\tau$. The algorithms developed here allow us to prove that a number of $E$-sequences with $a_{0}=7,8,9,10$ are nonrecurrent, verifying some of the conjectures in Galyean's thesis [9].

The paper is organized as follows: Section 1 recalls some notation from [4] and lists the additions to the list of small Salem numbers in [4]. Section 2 describes the basic algorithm for generating $S$ as adapted from [7]. In Section 3, given an interval $[\alpha, \beta]$, we modify the algorithm to deal with $S \cap[\alpha, \beta]$. If this set is finite, the algorithm detects this and determines all points in the set. Even if this set should contain a limit point $\theta \in S^{\prime}$, we show that it is possible to find all the points in the set provided the "width" of $\theta$ (see [5]) is sufficiently small. We describe a few items of interest from our catalogue of $S \cap[1,1.932]$, which includes the first six limit points of $S$. This list is complete except for a small interval around the limit point $\alpha_{3}=1.8667 \ldots$, but it is too long to reproduce here. In Section 4 we indicate how three of the new small Salem numbers were found.

In Section 5, we turn to the detailed study of Salem's construction. Given an interval $[\alpha, \beta]$ in the complement of $S \cup\{1\}$, we show how all $\theta_{m}^{ \pm}$in $[\alpha, \beta]$ can be determined provided $m \geqslant 2$. As an application we treat $m=2$ and $[\alpha, \beta]=[1.125,1.3]$, finding 41 Salem numbers. Only one of these had not been found previously. The fact that two of the 43 known Salem numbers in this interval are not of the form $\theta_{2}^{ \pm}$ shows that some members of $T$ can only be represented in the form $\theta_{1}^{ \pm}$. This shows that there can be no easy proof that inf $T>1$ by using the representation of $T$ as $\left\{\theta_{m}^{ \pm}: \theta \in S, m \geqslant 1\right\}$.

Section 6 describes a brief survey of $\theta$ in $S$ which have norm $\pm u_{0}$ and lie in $\left[u_{0}\right.$, $u_{0}+\delta$ ] for small $\delta$. A result communicated to me by C. J. Smyth [15] suggests that this may be an interesting way to find new small Salem numbers.

In Section 7, we give the application to Pisot sequences mentioned above. This requires investigating $S \cap[\alpha, \beta]$ for certain very small intervals $[\alpha, \beta]$. Finally, in Section 8 , we describe one of the many large finite families of $S$ and $T$ numbers discovered in the course of this investigation.

1. Notation. The set of minimal polynomials for elements of $S, T$ is denoted $S$, $T$, respectively. For polynomials of large degree we will often abbreviate

$$
a_{0} z^{k}+a_{1} z^{k-1}+\cdots+a_{k} \equiv a_{0} a_{1} \cdots a_{k} .
$$

We shall write $F_{m}$ for the cyclotomic polynomial whose roots are the primitive $m$ th roots of unity.

The Salem numbers $\leqslant 1.3$ found in [4] will be denoted $\sigma_{1}, \ldots, \sigma_{39}$ and their minimal polynomials $T_{1}, \ldots, T_{39}$. (There is a misprint in [4, p. 326]: $\sigma_{37}$ should be 1.2920391060 , not 1.2920391602 .) In addition to these, we have subsequently found

$$
\begin{aligned}
\sigma_{21.5} & =1.2767796740, & & \text { degree 22, } \\
\sigma_{40} & =1.2964213652, & & \text { degree 22, } \\
\sigma_{41} & =1.2968213737, & & \text { degree 28, } \\
\sigma_{42} & =1.2997448695, & & \text { degree 26, }
\end{aligned}
$$


with

$$
\begin{aligned}
T_{21.5} & =1-1-11000000-1010-10000001-1-11, \\
T_{40} & =1-1000-1000001000000-1000-11, \\
T_{41} & =1000-1-1-1-1-10000111111000-1-1-1-1-10001, \\
T_{42} & =1-1-1020-2-122-2-20030-2-222-1-2020-1-11 .
\end{aligned}
$$

2. The Basic Algorithm. Given $\theta \in S$, let $P$ be its minimal polynomial of degree $s$, and let $Q(z)=z^{s} P\left(z^{-1}\right)$. Then $Q$ has $Q(0)=1$, integer coefficients and exactly one zero in $|z|<1$, namely $\theta^{-1}$. If $A(z)$ is a polynomial with integer coefficients, not identical with $Q$, having $A(0)>0$ and $|A(z)| \leqslant|Q(z)|$ for $|z|=1$, then $f=A / Q$ is a rational function said to be associated with $\theta$. For $A$, one can take $A=(\operatorname{sgn} P(0)) P$, unless $Q(z)=1-q z+z^{2}$, in which case $A(z) \equiv 1$ is a suitable choice. Denote the set of such $f$ by $C$. Then $C$ is characterized by the following properties [7], [13] :

(i) $f$ is holomorphic in $|z| \leqslant 1$ except for a simple pole at $\theta^{-1}<1$,

(ii) $|f(z)| \leqslant 1$ on $|z|=1$,

(iii) $f(z)=u_{0}+u_{1} z+\cdots$ for $|z|<\theta^{-1}$, where the $u_{i}$ are integers, and $u_{0} \geqslant 1$.

The set $\mathcal{C}$ can be given the topology of convergence on compact subsets of the sphere, and then the subset of $C$ corresponding to a bounded set of $\theta$ is compact.

In [7], Dufresnoy and Pisot showed that the coefficient sequence $\left\{u_{n}\right\}$ of a member of $C$ is characterized by the following recursive system of inequalities:

$$
\begin{aligned}
1 & \leqslant u_{0}, \\
u_{0}^{2}-1 & \leqslant u_{1}, \\
w_{n}\left(u_{0}, \ldots, u_{n-1}\right) & \leqslant u_{n} \leqslant w_{n}^{*}\left(u_{0}, \ldots, u_{n-1}\right), \quad \text { for } n \geqslant 2 .
\end{aligned}
$$

The inequalities $w_{n} \leqslant u_{n} \leqslant w_{n}^{*}(n \geqslant 2)$ restrict $u_{n}$ to a finite interval except in one case: if $u_{0}=1$, then $w_{2}^{*}=\infty$.

To determine $w_{n}$ and $w_{n}^{*}$ one proceeds as follows. Let $D_{n}$ be a polynomial of the form $D_{n}=-z^{n}+d_{1} z^{n-1}+\cdots+d_{n}$ and let $E_{n}(z)=-z^{n} D_{n}\left(z^{-1}\right)$, so $E_{n}(0)=1$. Then $d_{1}, \ldots, d_{n}$ can be determined so that the first $n$ coefficients $u_{0}, \ldots, u_{n-1}$ of the expansion of $D_{n} / E_{n}$ match those of $f$, and then

$$
D_{n}(z) / E_{n}(z)=u_{0}+\cdots+u_{n-1} z^{n-1}+w_{n}\left(u_{0}, \ldots, u_{n-1}\right) z^{n}+\cdots .
$$

Similarly, let $D_{n}^{*}(z)=z^{n}+d_{1}^{*} z^{n-1}+\cdots+d_{n}^{*}$ and $E_{n}^{*}(z)=z^{n} D_{n}^{*}\left(z^{-1}\right)$, where $d_{1}^{*}, \ldots$, $d_{n}^{*}$ are determined so that

$$
D_{n}^{*}(z) / E_{n}^{*}(z)=u_{0}+\cdots+u_{n-1} z^{n-1}+w_{n}^{*}\left(u_{0}, \ldots, u_{n-1}\right) z^{n}+\cdots .
$$

The polynomials are conveniently determined from the relations

$$
\begin{aligned}
& D_{n+1}(z)=(1+z) D_{n}(z)-\left(u_{n}-w_{n}\right)\left(u_{n-1}-w_{n-1}\right)^{-1} z D_{n-1}(z), \\
& D_{n+1}^{*}(z)=(1+z) D_{n}^{*}(z)-\left(w_{n}^{*}-u_{n}\right)\left(w_{n-1}^{*}-u_{n-1}\right)^{-1} z D_{n-1}^{*}(z) .
\end{aligned}
$$

Equality holds in $w_{n} \leqslant u_{n}$ only if $f=D_{n} / E_{n}$, and then the $w_{m}=w_{m}^{*}$ for $m \geqslant n+1$ are simply the coefficients of $D_{n} / E_{n}$. Similarly, $u_{n}=w_{n}^{*}$ only if $f=D_{n}^{*} / E_{n}^{*}$. Note 
that $D_{n}, D_{n}^{*}$, in general, have rational coefficients only, not necessarily integer coefficients.

The sequences of integers $\left\{u_{n}\right\}$ satisfying (1) form an infinite tree, as described in [4]. A slight modification of that tree will be used here. The nodes of the tree at height $n$ are certain finite sequences $\left(u_{0}, \ldots, u_{n}\right)$ : there is a node at height -1 corresponding to the empty sequence; its successors, the nodes at height 0 are $\left(u_{0}\right)$, for all integers $u_{0} \geqslant 1$; if $\left(u_{0}, \ldots, u_{n}\right)$ is a node at height $n$ and $w_{n}<u_{n}<w_{n}^{*}$, then its successors are the nodes $\left(u_{0}, \ldots, u_{n}, u_{n+1}\right)$ for $w_{n+1} \leqslant u_{n+1} \leqslant w_{n+1}^{*}$ (if any such $u_{n+1}$ exist), while if $u_{n}=w_{n}$ or $u_{n}=w_{n}^{*}$, then $\left(u_{0}, \ldots, u_{n}\right)$ has no successors. A node without successors is called a terminal node. These are of three types: (i) $w_{n}<$ $u_{n}<w_{n}^{*}$, but there are no integers in $\left[w_{n+1}, w_{n+1}^{*}\right]$, (ii) $u_{n}=w_{n}$ (or $w_{n}^{*}$ ), but $D_{n}$ (or $D_{n}^{*}$ ) does not have integer coefficients, (iii) $u_{n}=w_{n}$ (or $w_{n}^{*}$ ), and $D_{n}$ (or $D_{n}^{*}$ ) has integer coefficients. The third type of terminal node is in one-one correspondence with the points of $S$, excluding the reciprocal quadratics $\left(q+\left(q^{2}-4\right)^{1 / 2}\right) / 2$, for $q \geqslant 3$.

Note that, for $n \geqslant 2$, the valence of the node $\left(u_{0}, \ldots, u_{n}\right)$ is finite. A path to infinity in this tree corresponds to an infinite sequence $\left\{u_{n}\right\}$ which satisfies $w_{n}<u_{n}$ $<w_{n}^{*}$ for all $n$, and hence to an $f=A / Q$ with $|A(z)|<|Q(z)|$ except at a finite number of points on $|z|=1$ [7]. Such $f$ are in the derived set $C^{\prime}$ and correspond to points $\theta \in S^{\prime}$.

In our computer implementation of the above, we do not use $D_{n}, D_{n}^{*}$ but rather

$$
\begin{aligned}
& F_{n}(z)=f_{n, 0} z^{n}+\cdots+f_{n, n}, \\
& F_{n}^{*}(z)=f_{n, 0}^{*} z^{n}+\cdots+f_{n, n}^{*},
\end{aligned}
$$

where $f_{n, 0}, \ldots, f_{n, n}$ are integers with no common divisor, and where $D_{n}=-F_{n} / f_{n, 0}$, and similarly for $F_{n}^{*}$. Then $w_{n}=W_{n} / f_{n, 0}$ and $w_{n}^{*}=W_{n}^{*} / f_{n, 0}^{*}$. The recursion for $F_{n}$, $F_{n}^{*}$ and formulas for $W_{n}, W_{n}^{*}$ are easily derived from (2)-(5).

Since the sequence $\left\{u_{n}\right\}$ grows exponentially, multiprecision arithmetic is essential. We made use of a quadruple precision decimal integer package supplied by the University of British Columbia Computing Centre. The use of fixed precision limits the height of the nodes which can be represented, but other factors prove to be critical in most cases.

It should be pointed out that it is not strictly necessary to calculate both $F_{n}$ and $F_{n}^{*}$ at each stage since one may use

$$
w_{n+1}^{*}-w_{n+1}=4\left(w_{n}^{*}-u_{n}\right)\left(u_{n}-w_{n}\right)\left(w_{n}^{*}-w_{n}\right)^{-1}
$$

as in [7]. However, we do want to know $F_{n}^{*}$ whenever $f_{n, 0}^{*}=1$, so it was found more convenient to compute both $F_{n}$ and $F_{n}^{*}$ and to determine $w_{n}, w_{n}^{*}$ directly from (2) and (3).

An interesting point is that in (4) and (5) only the differences $u_{n}-w_{n}$ and $w_{n}^{*}$ $-u_{n}$ arise, not $u_{n}$ itself. Thus, we really only need to be able to calculate $w_{n}$ (mod $f_{n, 0}$ ) and $w_{n}^{*}-w_{n}$, both of which are relatively small (though $f_{n, 0}$ can grow exponentially at a slower rate than $\left.u_{n}\right)$. However, a calculation of $w_{n}\left(\bmod f_{n, 0}\right)$ seems to require a knowledge of $u_{0}, \ldots, u_{n-1}\left(\bmod f_{n, 0}\right)$, and the least time-consuming way of 
determining these seems to be to have already computed $u_{0}, \ldots, u_{n-1}$. Otherwise, the advantage of the tree structure is lost.

Of course, we cannot traverse an infinite tree. In succeeding sections, we investigate conditions under which it suffices to search a finite subtree of the tree just described.

3. Pisot Numbers in an Interval. Let $[\alpha, \beta] \subset[1, \infty)$, and consider those $f$ in $C$ for which $\theta \in[\alpha, \beta]$. We shall see that, in addition to $(1),\left\{u_{n}\right\}$ must satisfy additional bounds

$$
v_{n}\left(\alpha ; u_{0}, \ldots, u_{n-1}\right) \leqslant u_{n} \leqslant v_{n}^{*}\left(\beta ; u_{0}, \ldots, u_{n-1}\right) .
$$

By [7], $D_{n}, D_{n}^{*}$ have roots $\tau_{n}, \tau_{n}^{*}$, such that $\tau_{n} \leqslant \tau_{n+1} \leqslant \theta \leqslant \tau_{n+1}^{*} \leqslant \tau_{n}^{*}$. If $\theta \leqslant \beta$ and $\theta \neq \tau_{n+1}$, then $D_{n-1}(\beta), D_{n}(\beta)$ and $D_{n+1}(\beta)$ are all $<0$, and by (4), this is equivalent to

$$
u_{n} \leqslant w_{n}+\left(u_{n-1}-w_{n-1}\right)(1+\beta) \beta^{-1} D_{n}(\beta) / D_{n-1}(\beta)=v_{n}^{*}(\beta) .
$$

By using (4) again, we can obtain a recursion for $v_{n}^{*}$ similar to (8). For, (4) gives

$$
\begin{aligned}
(1+\beta) \beta^{-1} D_{n+1}(\beta) / D_{n}(\beta) & \\
\quad= & (1+\beta)^{2} \beta^{-1}-\left(u_{n}-w_{n}\right)\left(u_{n-1}-w_{n-1}\right)^{-1}(1+\beta) D_{n-1}(\beta) / D_{n}(\beta) .
\end{aligned}
$$

Using the definition of $v_{n}^{*}$ from (10) and simplifying, we have

$$
v_{n+1}^{*}-w_{n+1}=(1+\beta)^{2} \beta^{-1}\left(v_{n}^{*}-u_{n}\right)\left(u_{n}-w_{n}\right) /\left(v_{n}^{*}-w_{n}\right) .
$$

Similarly, if $\theta \geqslant \alpha$, we use $D_{n}^{*}$ and (5) to obtain

$$
u_{n} \geqslant w_{n}^{*}-\left(w_{n-1}^{*}-u_{n-1}\right)(1+\alpha) \alpha^{-1} D_{n}^{*}(\alpha) / D_{n-1}^{*}(\alpha)=v_{n}(\alpha)
$$

And, as with $v_{n}^{*}$ we obtain

$$
w_{n+1}^{*}-v_{n+1}=(1+\alpha)^{2} \alpha^{-1}\left(w_{n}^{*}-u_{n}\right)\left(u_{n}-v_{n}\right) /\left(w_{n}^{*}-v_{n}\right) \text {. }
$$

The recurrence relations (12) and (14) must be supplemented with values for $v_{0}^{*}-w_{0}$ and $w_{0}^{*}-v_{0}$. Also, when $u_{0}=1$, we have $w_{2}^{*}=\infty$ so a value for $v_{2}(\alpha)$ must be calculated from the condition $D_{3}\left(u_{0}, u_{1}, v_{2}(\alpha) ; \alpha\right)=0$.

Note that $v_{n}(1)=w_{n}$ and $v_{n}^{*}(1)=w_{n}^{*}$, so that (12) and (14) reduce to (8) if $\alpha=$ $\beta=1$.

There is some difficulty in putting these formulas to immediate use since one cannot perform the indicated calculations exactly for arbitrary $\alpha$ and $\beta$. Even with $\alpha$ and $\beta$ rational it is not practical to use the required high precision arithmetic in order to perform the computations exactly. Our solution was to compute bounds $V_{n}(\alpha)$ and $V_{n}^{*}(\beta)$ satisfying $V_{n}(\alpha) \leqslant v_{n}(\alpha)$ and $v_{n}^{*}(\beta) \leqslant V_{n}^{*}(\beta)$. Here $V_{n}, V_{n}^{*}$ are rationals with a fixed denominator $d\left(=10^{7}\right.$ in most of our calculations). $V_{n}$ and $V_{n}^{*}$ are computed by the correctly rounded versions of (12) and (14). At a certain point $n=n_{0}$, the quadruple precision representation becomes inadequate; and then we shift to the simpler, but less precise, bounds described next. 
From Parseval's relation applied to $(1-\theta z) f(z)$ (cf. [14]), we have

$$
u_{0}^{2}+\left(u_{1}-u_{0} \theta\right)^{2}+\cdots \leqslant 1+\theta^{2}
$$

and hence, certainly

$$
\left(u_{n}-u_{n-1} \theta\right)^{2} \leqslant \theta^{2}
$$

so

$$
\left(u_{n-1}-1\right) \theta \leqslant u_{n} \leqslant\left(u_{n-1}+1\right) \theta .
$$

Thus, if $\alpha \leqslant \theta \leqslant \beta$, we must have

$$
\left(u_{n-1}-1\right) \alpha \leqslant u_{n} \leqslant\left(u_{n-1}+1\right) \beta .
$$

The tree that we should search is defined by

$$
\max \left(w_{n}, v_{n}(\alpha)\right) \leqslant u_{n} \leqslant \min \left(w_{n}^{*}, v_{n}^{*}(\beta)\right),
$$

with the conventions of Section 2 in case of equality. The tree that we in fact seek is defined by

$$
U_{n}(\alpha) \leqslant u_{n} \leqslant U_{n}^{*}(\beta)
$$

where

$$
\begin{aligned}
& U_{n}(\alpha)= \begin{cases}\max \left(V_{n}(\alpha), w_{n}\right), & \text { if } n<n_{0}, \\
\max \left(\left(u_{n-1}-1\right) \alpha, w_{n}\right), & \text { if } n \geqslant n_{0}\end{cases} \\
& U_{n}^{*}(\beta)= \begin{cases}\min \left(V_{n}^{*}(\beta), w_{n}^{*}\right), & \text { if } n<n_{0}, \\
\min \left(\left(u_{n-1}+1\right) \beta, w_{n}^{*}\right), & \text { if } n \geqslant n_{0} .\end{cases}
\end{aligned}
$$

Now, suppose that $S \cap[\alpha, \beta]$ is finite, hence contains no points of $S^{\prime}$. Then the tree defined by (19) has no paths to infinity, and each node has finite valence, hence the tree is finite by König's lemma [11, p. 381]. Thus, a search of this tree can be accomplished by any standard tree traversing algorithm; see [11, p. 334]. It is important to observe that the tree defined by (20) is also finite. For, if not, there would be an infinite sequence $u_{n}$ satisfying (20) for all $n$. However, $u_{n} / u_{n-1} \rightarrow \theta$, so $u_{n} \geqslant U_{n}(\alpha)$ $\geqslant\left(u_{n-1}-1\right) \alpha$ implies $\theta \geqslant \alpha$. Similarly, $\theta \leqslant \beta$. But this infinite sequence $\left\{u_{n}\right\}$ corresponds to a limit point of $S$, which is a two-sided limit point, hence $S \cap[\alpha, \beta]$ is infinite, contrary to assumption.

We shall discuss computations using (20) at the end of this section. First, we consider what can be done if $[\alpha, \beta]$ contains a limit point of $S$. Suppose $\theta \in S^{\prime}$, so it corresponds to one or more $f$ in $C^{\prime}$. Using (8), it can be shown [7] that as $n \rightarrow \infty$, $w_{n}^{*}-w_{n}$ decreases to a limit $2 w(f)$, where $w(f)$ is called the width of $f$ [5]. It can also be shown that $w_{n}^{*}-u_{n} \rightarrow w(f)$ and $u_{n}-w_{n} \rightarrow w(f)$. In [5], we obtained the formula

$$
w(f)=\theta^{2} \exp \left\{(2 \pi)^{-1} \int_{0}^{2 \pi} \log \left(1-\left|f\left(e^{i t}\right)\right|^{2}\right) d t\right\}
$$


and calculated $w(f)$ for all $f$ in $C^{\prime}$ corresponding to $\theta<2$, which had been determined by Amara [1]. In most of these cases $w(f)=1$. However, for $f=g_{3, n}=$ $(1-z)\left(1+z^{n}\right) /\left(1-2 z+z^{n}-z^{n+1}\right)$, for $n \geqslant 3$, we have $1.755 \ldots=w\left(g_{3,3}\right) \leqslant$ $w\left(g_{3, n}\right) \leqslant w\left(g_{3,5}\right)=1.987 \ldots$ and $\lim w\left(g_{3, n}\right)=1.908 \ldots$ Thus, with $w(\theta)$ defined as the maximum of $w(f)$ over $f$ having a pole at $\theta^{-1}$, we have $w\left(\alpha_{n}\right) \geqslant 1.755 \ldots$ for $n \geqslant 3$, where $\alpha_{n}>1$ solves $z^{n+1}-2 z^{n}+z-1=0$.

If $f$ is in $C^{\prime}$, then $w(f) \geqslant 1$, while if $f$ is not in $C^{\prime}$, eventually $w_{n}^{*}-w_{n}=0$ so in this case we define $w(f)=0$.

The significance of $w(f)$ can be seen from the following result:

Lemma 1. Suppose that $f \in C^{\prime}$ and that $w(f)<\left(1+(17)^{1 / 2}\right) / 4=1.2803 \ldots$. Then a sufficiently small neighbourhood of $f=A / Q$ contains only functions of the form $g=P_{m} / Q_{m}$, where $Q_{m}(z)=Q(z) \pm z^{m} A(z)$ and $P_{m}(z)= \pm z^{s} Q_{m}\left(z^{-1}\right)$, s being the degree of $Q_{m}$.

Proof. Regarding $f$ as the sequence $\left\{u_{n}\right\}$, a neighbourhood of $f$ consists of $g(z)=v_{0}+v_{1} z+\ldots$ for which $v_{n}=u_{n}$ for $n=0,1, \ldots, N$ for a suitable choice of $N$. Let then $g$ be such a function, with $v_{N+1}=u_{N+1}+a$, where $a \neq 0$. Since $w(f)$ $<2$, we may assume $N$ is so large that $w_{N+1}^{*}-u_{N+1}<2$ and $u_{N+1}-w_{N+1}<2$, so that $|a|=1$. Let $w_{n}, w_{n}^{*}$ correspond to the sequence $\left\{u_{n}\right\}$ and $w_{n}^{\prime}, w_{n}^{* \prime}$ correspond to $\left\{v_{n}\right\}$. Then (8) implies that

$$
\left(w_{N+1}^{*}-w_{N+1}\right)^{\prime}=4\left(w_{N}^{*}-u_{N}-a\right)\left(u_{N}+a-w_{N}\right) /\left(w_{N}^{*}-w_{N}\right) .
$$

Since $w_{N}^{*}-u_{N} \rightarrow w(f)$ and $u_{N}-w_{N} \rightarrow w(f)$, the right member of (23) tends to $2\left(w(f)^{2}-1\right) / w(f)<1$ since $w(f)<\left(1+(17)^{1 / 2}\right) / 4$. Thus, for sufficiently large $N$, the interval $\left[w_{N+1}^{\prime}, w_{N+1}^{* \prime}\right]$ is of length less than 1 . Because $w_{n}^{* \prime}-w_{n}^{\prime}$ is decreasing, the interval $\left[w_{n}^{\prime}, w_{n}^{* \prime}\right]$ will be of length less than 1 for $n \geqslant N+1$; and hence, there is at most one $g \in \mathcal{C}$ with expansion beginning $u_{0}, u_{1}, \ldots, u_{N}, v_{N+1}$. However, $P_{m} / Q_{m}$ is such an expansion for suitable $m$, the ambiguity in sign corresponding to the choices $a= \pm 1$.

Lemma 1, together with the previous discussion, shows that one can determine all of $S \cap[\alpha, \beta]$, even if $[\alpha, \beta]$ contains a limit point $\theta$ of $S$, provided $w(\theta)<1.2803 \ldots$. This explains how Dufresnoy and Pisot were able to go beyond the limit point $\tau=\alpha_{1}$, since $w(\tau)=1$.

To obtain an effective method, we need an estimate of $N$ in the proof of Lemma 1. We shall indicate such an estimate only if $w(f)<9 / 8=1.125$. In practice this is sufficient since, for all the known examples, either $w(f)=1$ or $w(f) \geqslant \theta_{0}^{2}=$ $1.7548 \ldots$ where $\theta_{0}=\min S$. If a conjecture of Lehmer is correct, then formula (25) of [5] for $w(f)$ shows that either $w(f) \leqslant 1$ or $w(f) \geqslant \sigma_{1}^{2}=1.3836 \ldots$ where $\sigma_{1}$ is the conjectured minimum of $T$.

LemMA 2. Let $f \in C^{\prime}$ have expansion $u_{0}+u_{1} z+\ldots$. Suppose that $w_{N}^{*}-w_{n} \leqslant$ $9 / 4$ for some $N$. Then, for any $n \geqslant N$, there are exactly two $g \in C$ with expansions beginning $u_{0}, \ldots, u_{n-1}, v_{n}$, with $v_{n} \neq u_{n}$.

Proof. As in Lemma 1 , let $g=u_{0}+\cdots+u_{n-1} z^{n-1}+v_{n} z^{n}+\ldots$ with $v_{n} \neq$ 
$u_{n}$; let $w_{n+1}^{\prime}, w_{n+1}^{* \prime}$ be as in that lemma. If $n \geqslant N$, then $w_{n}^{*}-w_{n} \leqslant 9 / 4$, so $v_{n}=$ $u_{n} \pm 1$ are the only possibilities. Let $a_{n}=u_{n}-w_{n}$ and $b_{n}=w_{n}^{*}-u_{n}$. Then $a_{n} \geqslant 1$, $b_{n} \geqslant 1$, since the choices $v_{n}=u_{n} \pm 1$ are both possible. We claim that, for $n \geqslant N$, and $\epsilon= \pm 1$,

$$
4\left(a_{n}-\epsilon\right)\left(b_{n}+\epsilon\right)<a_{n}+b_{n} .
$$

For, if $\epsilon=1$, then (24) is equivalent to

$$
b_{n}\left(4 a_{n}-5\right)+\left(3 a_{n}-4\right)<0
$$

which certainly holds if $a_{n} \leqslant 5 / 4$. However, $a_{n}=\left(w_{n}^{*}-w_{n}\right)-b_{n} \leqslant 9 / 4-1$ if $n \geqslant$ $N$. Similarly, for $\epsilon=-1$. Together, (24) and (8) imply that $\left(w_{n+1}^{*}-w_{n+1}\right)^{\prime}<1$ and, hence, that there is exactly one expansion which begins $u_{0}, u_{1}, \ldots, u_{n}, u_{n}+\epsilon$ for $\epsilon= \pm 1$, as in the proof of Lemma 1 .

Results. The first seven limit points of $S$, according to Amara [1], are

$$
\alpha_{1}=\beta_{1}<\alpha_{2}<\beta_{2}<\alpha_{3}<\hat{\theta}_{2}^{\prime}<\beta_{3}<\alpha_{4},
$$

where $\alpha_{n}$ solves $z^{n+1}-2 z^{n}+z-1=0$ and $\beta_{n}$ solves $z^{n+1}-z^{n}-\cdots-z-1=0$, while $\hat{\theta}_{2}^{\prime}$ solves $z^{4}-z^{3}-2 z^{2}+1=0$. Numerically, these are roughly

$$
1.618<1.755<1.839<1.867<1.905<1.928<1.933 \text {. }
$$

Only two of these have width exceeding 1 , namely $w\left(\alpha_{3}\right)=1.7548 \ldots$ and $w\left(\alpha_{4}\right)=$ $1.9635 \ldots$. Thus, it is possible by our methods to determine all points in $S \cap\left[1, \alpha_{3}\right.$ $-\epsilon]$ and in $S \cap\left[\alpha_{3}+\epsilon, \alpha_{4}-\epsilon\right]$ for any $\epsilon>0$. In fact, we used the algorithm to list all $\theta \in S \cap([1,1.86675] \cup[1.868,1.932])$ together with the corresponding $P \in$ $S$. The output is roughly the size of a small telephone directory so we shall only make a few remarks about it here. Dufresnoy and Pisot found that in the interval $\left[1, \hat{\theta}_{15}\right]$ $=[1,1.61836 \ldots]$, all but two of the points of $S$ are solutions of one of the equations $z^{n}\left(z^{2}-z-1\right) \pm\left(z^{2}-1\right)=0$ or $z^{n}\left(z^{2}-z-1\right) \pm 1=0$ corresponding to the two expansions $1 /\left(1-z-z^{2}\right)$ and $\left(1-z^{2}\right) /\left(1-z-z^{2}\right)$ in $C^{\prime}$. In addition there is the limit point $\tau$ which solves $z^{2}-z-1=0$, and one other $\phi$ which solves $z^{6}-2 z^{5}$ $+z^{4}-z^{2}+z-1=0$ which is best written in the form $z^{3}\left(z^{3}-2 z^{2}+z-1\right)+$ $(z-1)\left(z^{2}+1\right)=0$, and thus corresponds to one of the expansions associated with $\alpha_{2}$. From our list, the next polynomial which is not in one of the two infinite sequences above, is

$$
P=1-22-32-21001-12-22-21-1,
$$

which has $\theta=1.6216584885$, and was mentioned in [4] (we had found it by an earlier floating-point version of the algorithm described here). This is the only polynomial with $\theta$ in $[1,1.753]$ which has any coefficient whose absolute value exceeds 2 . In general, the coefficients of the polynomials for $\theta \in[1,1.932]$ were smaller than 4 in absolute value with only two polynomials having coefficients equal to 5 . One of these is

$$
P=1-22-43-54-55-55-44-33-22-11 \text {, }
$$

with $\theta=1.9055327245$. 
The neighbourhood of $\beta_{2}=1.839 \ldots$ produced some interesting results. Here there are two $f \in C^{\prime}$ with pole at $\beta_{2}^{-1}$, namely

and

$$
\left(1-z^{3}\right) /\left(1-z-z^{2}-z^{3}\right)=1123611 \ldots
$$

$$
(1+z) /\left(1-z-z^{2}-z^{3}\right)=12361120 \ldots,
$$

both of width 1 ; and hence by Lemma 1 , a sufficiently small neighbourhood of $\beta_{2}$ contains only solutions of $z^{n} P(z) \pm A(z)=0$, where $P(z)=z^{3}-z^{2}-z-1$ and $A(z)=$ $z^{3}-1$ or $z+1$. However, even in the small neighbourhood $(1.839,1.8396)$ there were 21 numbers not of this form. Among these were the roots of

and

$$
z^{n}\left(z^{3}-z^{2}-z-1\right)-1 \quad \text { for } n=12,13,15,16,18,21,24 \text { and } 27
$$

$$
z^{n}\left(z^{3}-z^{2}-z-1\right)+1 \text { for } n=11,12,14,17,20 \text { and } 23 .
$$

Since $1 /\left(1-z-z^{2}-z^{3}\right)$ exceeds 1 in absolute value on $|z|=1$, there can only be a finite number of such occurrences and our computation detects them all. Since

$$
1 /\left(1-z-z^{2}-z^{3}\right)=11247132444 \ldots \text {, }
$$

it is tempting to regard this phenomenon as being somehow due to the presence of the nearby $g_{3,3}$ with $w\left(g_{3,3}\right)>1$, since

$$
g_{3,3}=11247132445 \ldots .
$$

As an example of the computational time involved, the determination of the 165 Pisot numbers in $[1.755,1.839]$ took 20.4 seconds of CPU time on the IBM $370 / 165$ here, examining 2355 nodes of the appropriate tree.

4. Some New Small Salem Numbers. If $P \in S$ has degree $k$, with $\operatorname{root} \theta>1$, then Salem [13] showed that, for $\epsilon= \pm 1$,

$$
P_{m}^{\epsilon}(z)=z^{m} P(z)+\epsilon z^{k} P\left(z^{-1}\right)=0
$$

has a root $\theta_{m}^{\epsilon} \geqslant 1$ which is a Salem number for all sufficiently large $m$, provided $P$ is not a reciprocal quadratic. In fact, $\theta_{m}^{+} \in T$ for all $m$, while $\theta_{m}^{-} \in T$ if and only if $m>$ $k-2 P^{\prime}(1) / P(1)$. We showed in [4] that all Salem numbers arise in this way.

It is easily seen that $\theta_{m}^{\epsilon}<\theta$ if $\epsilon=\operatorname{sgn} P(0)$ so we will always make this choice of $\epsilon$ and write $\theta_{m}^{\epsilon}=\theta_{m}$ for simplicity. Then (26) can be rewritten in the form

$$
1+z^{m} f(z)=0
$$

where

$$
f(z)=\operatorname{sgn} P(0) P(z) / z^{k} P\left(z^{-1}\right),
$$

so $f \in C$. The equation (27) has at most one root in $|z|<1$, namely $\theta_{m}^{-1}$. This holds true for any $f \in C$ even when $f$ is not of the form (28). If $f$ is not of the form (28), then $f \in C^{\prime}$; and if $\theta_{m}>1$, then $\theta_{m} \in S$. If (27) has no root in $|z|<1$, we define $\theta_{m}=1$. It should be observed that $\theta_{m}$ depends on $f$ and not simply on the pole $\theta^{-1}$ of $f$.

In the computations described in Section 3, along with $\theta \in S$ and $P \in S$, we calculated the smallest corresponding $\theta_{m}>1$. From the $\theta \leqslant 1.932$, we found exactly 
one new $\theta_{m}<1.3$, namely $\sigma_{41}$ which is $\theta_{1}^{-}$for the degree $28 \theta=1.8388762951$ with

$$
P=1-21-1-11-1101001-1000-10000001-110-11-1 \text {. }
$$

In fact, $P_{1}^{-}(z)=(z-1) T_{41}(z)$.

By inspection, $T_{41}(z)\left(z^{8}+1\right)=z^{29} Q(z)+z^{7} Q\left(z^{-1}\right)$, where $Q=1000-1$ $-1-1-1$. The polynomial $Q$ has 3 roots in $|z|>1$, one at $\theta=1.296466239$ and a pair $\alpha, \bar{\alpha}$ with $|\alpha| \simeq 1.052$, so the method of [4] may be applied to find Salem numbers among the roots of $Q_{m}^{ \pm}(z)$. In fact, $Q_{37}^{-}=F_{1} F_{2} F_{4} F_{11} F_{16} T_{40}$, and this is the way $\sigma_{40}$ was first discovered.

The Salem number $\sigma_{21.5}$ was found in a similar way. We observe that $(z+1) T_{28}$ $=Q_{20}^{+}$for the degree $11 Q=1-100-11-110-11-1$. This $Q$ has five roots in $|z|>1$ at $\theta=1.2816970361$ and $\alpha, \bar{\alpha}, \beta, \bar{\beta}$ with $|\alpha| \simeq 1.035$ and $|\beta| \simeq 1.034$. We find that $Q_{19}^{-}=F_{1} F_{2} F_{3} F_{4} F_{6} T_{21.5}$.

5. Salem Numbers in Intervals. We now investigate Salem's construction in more detail. For each $m \geqslant 1$ and $f \in C$, let $\theta_{m}^{-1}$ be the root of (27) identified in Section 4. Let $D_{n}, D_{n}^{*}$ be the polynomials corresponding to $f$ as in Section 2. It is clear that $E_{n}(z)+z^{m} D_{n}(z)=0$ has at most one root, say $\tau_{n, m} \geqslant 1$ in $|z|>1$. If no such root exists, write $\tau_{n, m}=1$. The equation $E_{n}^{*}(z)+z^{m} D_{n}^{*}(z)=0$ has exactly one root $\tau_{n, m}^{*}$ $>1$ in $|z|>1$. Furthermore, we have the following:

LEMMA 3. The following inequality holds for all $m \geqslant 1$ :

$$
\tau_{n, m} \leqslant \theta_{m} \leqslant \tau_{n, m}^{*}
$$

Proof. Let $f$ be as above, and let $\alpha=\theta^{-1}$. The function

$$
\left(E_{n}(z) f(z)-D_{n}(z)\right)(z-\alpha)
$$

is holomorphic in $|z| \leqslant 1$ and, if not identically zero, is negative for $0<z<1$, as shown in $[7$, p. 80]. Thus

$$
\begin{aligned}
\left\{E_{n}\left(1+z^{m} f\right)-\left(E_{n}+z^{m} D_{n}\right)\right\}(z-\alpha) \\
=z^{m}\left(E_{n} f-D_{n}\right)(z-\alpha)<0 \text { for } 0<z<1 .
\end{aligned}
$$

Let $\psi=\tau_{n, m}^{-1}$. It is clear that $\alpha=\theta^{-1} \leqslant \tau_{n}^{-1}<\tau_{n, m}^{-1}=\psi \leqslant 1$. If $\psi<1$, we can set $z=\psi$ in (29) and obtain

$$
E_{n}(\psi)\left(1+\psi^{m} f(\psi)\right)(\psi-\alpha)<0 .
$$

Now $E_{n}$ vanishes once in $0<z<1\left(\right.$ at $\left.\tau_{n}^{-1}\right)$ and $E_{n}(0)=1>0$, so $E_{n}(\psi)<0$. Thus (30) implies $\left(1+\psi^{m} f(\psi)\right)(\psi-\alpha)>0$. However, at $z=0$, we clearly have $\left(1+0^{m} f(0)\right)(0-\alpha)<0$. Hence $z^{m} f(z)+1$ vanishes in $0<z<\psi$, so $\theta_{m}>\tau_{n, m}$. Of course, if $\psi=1$, the result $\theta_{m} \geqslant 1=\tau_{n, m}$ is trivial.

The proof that $\theta_{m} \leqslant \tau_{n, m}^{*}$ is similar.

We now consider the following problem: given $m \geqslant 1$ and an interval $[\alpha, \beta]$, determine all $\theta_{m}$ in $[\alpha, \beta]$. We shall see that this problem can be solved effectively for $m \geqslant 2$, provided $[\alpha, \beta]$ does not intersect $\{1\} \cup S$.

The restriction $\alpha>1$ may seem unfortunate since we are interested in the 
question of whether inf $T>1$. However, if one could determine an interval $[\alpha, \beta]$ with $1<\alpha<\beta$ such that $[\alpha, \beta] \cap T$ is empty then, since $T$ is closed under the operation of taking successive powers, one would also know that $\left[\alpha^{1 / k}, \beta^{1 / k}\right] \cap T$ is empty, for all $k=1,2, \ldots$ If $1 / m<(\log \beta / \log \alpha)-1$, then $\bigcup_{k=m}^{\infty}\left[\alpha^{1 / k}, \beta^{1 / k}\right]=\left(1, \beta^{1 / m}\right]$, and one would have inf $T \geqslant \beta^{1 / m}$.

By a similar argument, if one could determine an interval $[\alpha, \beta]$ with $\alpha<\beta^{1 / 2}$ such that $[\alpha, \beta] \cap T$ is a finite set, then one could determine all points in $(1, \beta] \cap T$ and, hence, find $\min T$. For each fixed $k$ it is certainly clear that one can determine for each $\sigma \in[\alpha, \beta] \cap T$ whether or not $\sigma^{1 / k} \in T$ (by checking a finite number of polynomials). Only a finite number of values of $k$ would need to be checked since the degree of $\sigma \in T$ increases at an estimabie rate as $\sigma \rightarrow 1$, by the work of Blanksby and Montgomery [2].

Returning to our main problem, we wish to consider $f \in \mathcal{C}$ with expansion $u_{0}+$ $u_{1} z+\cdots$ and to determine conditions on $\left\{u_{n}\right\}$ so that $\theta_{m} \in[\alpha, \beta]$. Fix $m$, and let $B_{n}(z)=z^{m} D_{n}(z)+E_{n}(z)$. It can be shown that $B_{n}$ satisfies the recurrence relation (4). If we are to have $\theta_{m} \leqslant \beta$, then by Lemma $3, \tau_{n+1, m} \leqslant \beta$, and this means $B_{n+1}(\beta)$ $\leqslant 0$. Using (4) just as in Section 2 , we find that this implies a condition $u_{n} \leqslant s_{n}^{*}(\beta)$, where $s_{n}^{*}$ satisfies the recurrence relation

$$
s_{n+1}^{*}-w_{n+1}=(1+\beta)^{2} \beta^{-1}\left(s_{n}^{*}-u_{n}\right)\left(u_{n}-w_{n}\right) /\left(s_{n}^{*}-w_{n}\right) .
$$

The only explicit dependence on $m$ is through the initial condition, and we find that

$$
s_{0}^{*}(\beta)=\left(\beta^{m+1}-1\right) /\left(\beta^{m}-\beta\right)
$$

Note that when $m=1$ we have $s_{0}^{*}(\beta)=\infty$, which, if used in (31) gives

$$
s_{1}^{*}-w_{1}=(1+\beta)^{2} \beta^{-1}\left(u_{0}+1\right) \text {. }
$$

This can be verified to be the correct value.

Similarly, using $\alpha^{m} D_{n}^{*}(\alpha)+E_{n}^{*}(\alpha)<0$, we find a condition $u_{n} \geqslant s_{n}(\alpha)$, where

$$
w_{n+1}^{*}-s_{n+1}=(1+\alpha)^{2} \alpha^{-1}\left(w_{n}^{*}-u_{n}\right)\left(u_{n}-s_{n}\right) /\left(w_{n}^{*}-s_{n}\right)
$$

and

$$
s_{0}(\alpha)=-\left(\alpha^{m+1}+1\right) /\left(\alpha^{m}+\alpha\right)
$$

Again, if $u_{0}=1$, the fact that $w_{2}^{*}=\infty$ means that we must use a special formula for $s_{2}(\alpha)$.

Lemma 4. Suppose $[\alpha, \beta]$ does not intersect the set $\{1\} \cup S$. Let $P$ be the tree of Section 2 restricted by the further conditions

$$
s_{n}(\alpha) \leqslant u_{n} \leqslant s_{n}^{*}(\beta), \text { for all } n \geqslant 0
$$

If $m \geqslant 2$, then $P$ is a finite tree. If $m=1$, and $u_{0}$ is fixed, then the tree defined by the remaining conditions is finite.

Proof. If not, $P$ would contain a path to infinity which would correspond to an $f \in C^{\prime}$. For this $f$ we would have $\theta_{m}=1$ or $\theta_{m} \in S$. However, the inequalities (36) 
guarantee that $\alpha \leqslant \theta_{m} \leqslant \beta$, which contradicts the assumption that $[\alpha, \beta]$ does not inter$\operatorname{sect}\{1\} \cup S$.

To put these results into practice, similar remarks to those concerning $v_{n}$ and $v_{n}^{*}$ in Section 2 may be made. That is, we in fact replace (36) by

$$
S_{n}(\alpha) \leqslant u_{n} \leqslant S_{n}^{*}(\beta) \text {, }
$$

where $S_{n}, S_{n}^{*}$ are rational numbers with a fixed denominator satisfying $S_{n} \leqslant s_{n}$ and $s_{n}^{*} \leqslant S_{n}^{*}$. Unfortunately, no auxiliary inequalities such as (19) seem to be available here, so it is possible that the tree defined by (37) may be infinite even when the tree defined by (36) is finite. This could occur if, e.g., $\left[S_{n}\right]<\left[s_{n}\right]$ at some stage. This, in fact, caused some difficulty in practice when the denominator of $S_{n}, S_{n}^{*}$ was chosen to be $d=10^{3}$, but the choice of $d=10^{7}$ avoided this problem in all the computations to be described next.

We now describe the application of this algorithm to find all Salem numbers of the form $\theta_{m}$ for $m \geqslant 2$ in certain intervals $[\alpha, \beta]$ with $\beta \leqslant 1.3$. By the results of [4, p. 322], if $\theta>\gamma_{m}(x)=\left(x^{m+1}-1\right) /\left(x^{m}-x\right)$, then $\theta_{m}>x$. Taking $x=1.3$, we find that, for $m=2,3,4,5$ we have $\gamma_{m}(1.3)=3.069,2.069,1.743,1.586$. From our list of $S \cap[1,1.86675]$, we can thus treat $m \geqslant 4$ by inspection. In fact, $m \geqslant 6$ was already discussed in [4]. The number of $P \in S$ which produce $\theta_{m} \in(1,1.3]$ is $1,1,2$, 2,6 for $m=8,7,6,5,4$, respectively.

For $m=3$, we could not deal with the entire interval $(1,1.3]$, but restricted our search to $[1.1,1.3]$. The tree which was searched had 317 nodes, height 28 , and $22 P$ were found for which $\theta_{3} \in[1.1,1.3]$, all of degree at most 16 .

For $m=2$, we examined an even smaller interval [1.125,1.3] in two separate parts $[1.125,1.2]$ and $[1.2,1.3]$. For $[1.2,1.3]$, the tree had height 57 , had 42499 nodes and 544 suitable $P$ were found. For $[1.125,1.3]$, there were correspondingly 14603 nodes, height 38 and $32 P$. The total CPU time for these various calculations was about 17 minutes. Most of the time was spent in searching the subtree for which $\left(u_{0}, \ldots, u_{4}\right)=(1,2,4,8,16)$. The reason for this is readily seen: the function $1 /(1-2 z)=1+2 z+4 z^{2}+\cdots$ is in $C^{(2)}$, the second derived set of $C$, and has $\theta_{2}=$ 1. Thus, a neighbourhood of this function contains a great many $g \in \mathcal{C}$ for which $\theta_{2}$ is close to 1 . All such $\theta_{2}$ which satisfy $\theta_{2} \geqslant \alpha$ must occur in the search of the tree.

The program contained a provision for recognizing already known Salem numbers and factoring the polynomials $P_{m}$ which occurred. For example, the largest degree polynomial found was of degree 46 , namely

$$
P(z)=z^{46}-2 z^{45}-z^{39}-z^{34}-z^{29}-z^{22}-z^{16}-z^{11}-z^{6}-1,
$$

with $\theta=2.0153852503$ and $P_{2}^{-}=F_{1} F_{2} F_{10} F_{46} T_{37}$.

The new small Salem number $\sigma_{42}$ of degree 26 appeared twice on this list. There are thus 43 known Salem numbers in $[1.125,1.3]$. The number of times each appears in the list of $576=544+32$ numbers just mentioned is roughly correlated with the degree of the number. Only two numbers $\sigma_{23}$ (degree 26) and $\sigma_{31}$ (degree 44) did not appear. The numbers $\sigma_{11}, \sigma_{28}, \sigma_{29}, \sigma_{32}, \sigma_{33}$ of degrees $26,30,30,30,34$ each 
appeared once only, while $\sigma_{22}$ (degree 8) appeared 67 times and $\sigma_{38}$ (degree 10) appeared 68 times.

6. Intervals Near Integers. In [4], it was shown that if $|P(0)|=u_{0}$, and $\theta>$ $u_{0}+2+x+x^{-1}$, then $\theta_{1}^{\epsilon}>x$, so that small Salem numbers can only be produced in this case for $u_{0} \leqslant \theta \leqslant u_{0}+2+x+x^{-1}$. C. J. Smyth [15] has proved a sort of converse of this, which states that if $\theta_{1}^{-}>1$ and $\theta<u_{0}+\epsilon^{2}$ with $0<\epsilon<1 / 2$, then $\theta_{1}^{-}<1 /(1-\epsilon)$. His interesting proof considers the conjugates of $\theta$ in detail. The following is a comparable result which instead uses Lemma 3.

Lemma 5. Let $|P(0)|=u_{0}>1, \epsilon=\operatorname{sgn} P(0)$ and $\sigma=\theta_{1}^{\epsilon}$. Suppose that $\theta=$ $u_{0}+\delta$. Then

$$
\sigma+\sigma^{-1}<2+\delta\left(u_{0}+1\right)^{2} /\left(u_{0}^{2}-u_{0}\right) .
$$

Hence, $\sigma<1+a \delta^{1 / 2}$ for a computable constant a.

Proof. By Lemma 3, $\sigma \leqslant \tau_{2,1}^{*}$. Now $D_{n}^{*}(z)=z^{2}-u_{1} /\left(u_{0}-1\right) z+u_{0}$, so $\tau_{2,1}^{*}$ solves $B(z)=0$ where $B(z)=\left(z D_{2}^{*}(z)+E_{2}^{*}(z)\right) /(z+1)=z^{2}-\left\{u_{1} /\left(u_{0}-1\right)-\right.$ $\left.\left(u_{0}-1\right)\right\} z+1$. Thus, we must have $0<B(\sigma)$ which reduces to

$$
\sigma+\sigma^{-1}<u_{1} /\left(u_{0}-1\right)-\left(u_{0}-1\right)
$$

On the other hand, by [7], we have $\tau_{2} \leqslant \theta$, which implies that $D_{2}(\theta) \leqslant 0$. Since $D_{2}(z)=-z^{2}+\left(u_{1} /\left(u_{0}+1\right)\right) z+u_{0}$, this implies

$$
u_{1} /\left(u_{0}+1\right) \leqslant\left(\theta^{2}-u_{0}\right) / \theta \text {. }
$$

Estimating $u_{1}$ in (39) by (40), and using $\left(1+\delta / u_{0}\right)^{-1}>1-\delta / u_{0}$ gives (38).

The supposition that inf $T>1$ thus has interesting consequences for the subset of $S$ satisfying the conditions of Lemma 5 , since $\theta_{1}^{\epsilon}>1$ implies $\theta_{1}^{\epsilon} \in T$. In particular, $\theta_{1}^{+}$is always in $T$. For example, if inf $T>1$, then there must be a lower bound $u_{0}$ $+\delta_{0}$ with $\delta_{0}>0$ independent of $u_{0}$ on those $\theta \in S$ satisfying $P(0)=u_{0}>0$. If $\sigma_{1}$ is indeed the smallest Salem number, then Lemma 5 gives $\delta_{0} \geqslant 0.026$.

A brief study was thus made of $S_{u} \cap[u, u+\delta]$ for small $\delta$, where $S_{u}=\{\theta \in$ $S: \theta^{-1}$ is the pole of an $f \in C$ with $f(0)=u$ \}. Note that $S_{u}$ is a closed set. It is easy to show, using the methods of [7], that $\min S_{u}$ is the root $\theta>1$ of the cubic $z^{3}-(u-1) z^{2}-u z-u$, so that $\min S_{u} \simeq u+(u+1)^{-1}$. Note that $\theta_{1}^{-}=1$. The smallest limit point appears to be $\left(u+\left(u^{2}+4\right)^{1 / 2}\right) / 2$ corresponding to $f(z)=$ $u /\left(1-u z-z^{2}\right)$. Since the size of this number is $u+u^{-1}-u^{-3}+O\left(u^{-5}\right)$, we see that a great many points of $S_{u}$ are to be found rather close to $\min S_{u}$.

For example, $\min S_{9}=9.0979635508$ and $\min S_{9}^{\prime} \leqslant 9.1097722286$. The interval $[9,9.1] \cap S_{9}$ contains 27 points, none with $P(0)>0$. An incomplete search of $[9.11,9.12] \cap S_{9}$ revealed the degree 16

$$
D_{16}^{*}=1-8-9-10-2-3555-4-5-621999,
$$

with $\theta=9.1112415981$ for which $\theta_{1}^{+}=\sigma_{1}$.

It is clear that the sets $S_{u}$ deserve a closer examination, but this will not be done here. 
7. Pisot Sequences. In [3] we proved that there are Pisot sequences which satisfy no linear recurrence; in particular $E(14,23)$ is such a sequence. After [3] was written, D. G. Cantor sent me a copy of the thesis of P. Galyean [9], in which many $E$-sequences with $a_{0} \leqslant 10$ were investigated. He found many which satisfied no recurrence of degree at most $d$ where typically $d=15,20,25$ or 20 . He conjectured that at least some of these in fact satisfy no recurrence whatsoever. We shall show that this in indeed the case for a number of his examples.

If $E\left(a_{0}, a_{1}\right)$ is an $E$-sequence, then $\lim a_{n+1} / a_{n}=\theta\left(a_{0}, a_{1}\right)=\theta$. If $E\left(a_{0}, a_{1}\right)$ is recurrent then it satisfies either an $S$-recurrence, so $\theta \in S$, or a $T$-recurrence, so $\theta \in T$. To show that an $S$-recurrence is not satisfied, it suffices then to show that $\theta \notin S$. The same idea was used in [3] but now our algorithm for deciding $\theta \notin S$ is more efficient.

The main contribution of [3] was a method for dealing with $T$-recurrence. Theorem 1 of [3] can handle most of the examples we discuss here, but a sharpening of that criterion is necessary for some. Unexplained notation in the following is taken from [3].

Let $E\left(a_{0}, a_{1}\right)$ be assumed for the moment to satisfy a $T$-recurrence. We call a recurrence pure if the generating function $f(z)=A(z) / B(z)$ has $\operatorname{deg} A<\operatorname{deg} B$. It is shown in [3] that if $\theta\left(a_{0}, a_{1}\right) \geqslant 2$, then the recurrence for $E\left(a_{0}, a_{1}\right)$ is pure. If $\tau=$ $\left(1+5^{1 / 2}\right) / 2<\theta<2$, then the recurrence is pure, provided $a_{0}>2+13 /\left(\theta^{2}-\theta-1\right)$; and this can always be arranged by dropping a few initial terms of the sequence. If $\theta$ $<\tau$, we cannot deal with general $T$-recurrence, but must assume, a priori, that the recurrence is pure.

Thus, let $E\left(a_{0}, a_{1}\right)$ satisfy a pure $T$-recurrence. Then $a_{n}$ has the form

$$
a_{n}=\lambda \theta^{n}+\mu \theta^{-n}+\delta_{n}, \quad n=0,1, \ldots,
$$

where $\theta$ and $\lambda$ can be computed to arbitrary accuracy and $\delta_{n}$ is an almost periodic sequence which satisfies

$$
\left|\theta^{2} \delta_{n}-2 \theta \delta_{n+1}+\delta_{n+2}\right| \leqslant 1 / 2 \text { for } n=0, \pm 1, \pm 2, \ldots .
$$

Using (41) as the definition of $a_{n}$ for $n<0$, it can be shown that the $a_{n}$ are integers. This fact, combined with (42) shows that the $a_{n},-K \leqslant n<0$ can be computed from a knowledge of $a_{0}, a_{1}, \ldots, a_{N}$ (where $K \rightarrow \infty$ as $N \rightarrow \infty$ ), provided $\theta>\tau$. The reason for the condition $\theta>\tau$ is that it implies $(1+\theta) / 2 \theta^{2}<1 / 2$ so that the $a_{n}$ are uniquely determined from

$$
\left|a_{n}-\Delta_{\theta}\left(a_{n+1}, a_{n+2}, a_{n+3}\right)\right| \leqslant(1+\theta) / 2 \theta^{2} .
$$

However, even if $1<\theta<\tau$, we still have $(1+\theta) / 2 \theta^{2}<1$, so the $a_{n}$, for $n<0$, form a tree which is at most binary. For example, $E(7,11)$ has $\theta(7,11)=1.5499304464<\tau$. If we assume that $E(7,11)$ satisfies a pure $T$-recurrence, then $a_{n}$ is given by $4,2,1,1$, $2,4,7$ for $n=-1, \ldots,-7$, while $a_{-8}=11$ or 12 . The next branching occurs at $a_{-23}$.

To state our new criterion, let $\nu=\mu\left(\theta-\theta^{-1}\right)^{2}$. Then (41) and (42) show that $\left|\nu-\left(a_{n+2}-2 \theta a_{n+1}+\theta^{2} a_{n}\right) \theta^{n}\right| \leqslant \theta^{n} / 2$ for all $n$. This restricts $\nu$ to an interval $I_{n}$ of length $\theta^{n}$ for each $n$, positive or negative. If $E\left(a_{0}, a_{1}\right)$ is $T$-recurrent, these intervals 
must have a nonempty intersection. For example, $E(9,15)$ is not $T$-recurrent since $I_{-1} \cap I_{-20}=\varnothing ; E(7,11)$ is not pure $T$-recurrent since $I_{0} \cap I_{-7}=\varnothing$. Furthermore, our list of $S$ shows $\theta(7,11) \notin S$, and $\theta(9,15)=1.6913056849 \notin S$. Hence, $E(9,15)$ satisfies no recurrence whatsoever, and $E(7,11)$ satisfies no pure recurrence.

For $\theta$ larger than 1.932, we apply the algorithm of Section 3 to a small interval $[\alpha, \beta]$ with $\alpha<\theta<\beta$. Continued fractions are used to select $\alpha$ and $\beta$ with small denominators. For example, to check that $\theta(7,15)=2.129112585158341 \notin S$, we looked at the interval

$[96617 / 45379,28215 / 13252]=\left[\begin{array}{lllll}2.1291125851 & 16,2.1291125867 & 79\end{array}\right]$, and found that $S \cap[\alpha, \beta]$ contains exactly one number $\theta$, of degree 41 , namely $\theta=$ 2.129112585158442 , with

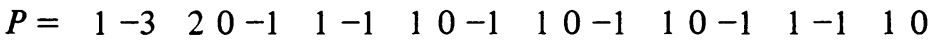

$$
\begin{aligned}
& \begin{array}{llllllllllllllllllllll}
-2 & 3 & -2 & 0 & 1 & -1 & 1 & -1 & 0 & 1 & -1 & 0 & 1 & -1 & 0 & 1 & -1 & 1 & -1 & 0 & 1 & -1 .
\end{array}
\end{aligned}
$$

We can thus conclude that $\operatorname{dist}(\theta(7,15), S)=1.011 \times 10^{-13}$.

Using these techniques, $E\left(a_{0}, a_{1}\right)$ has been proved to be nonrecurrent for $\left(a_{0}, a_{1}\right)$ $=(7,15),(8,14),(9,15),(9,17),(9,19),(10,18),(10,21)$ and $(10,22)$. In addition, the following do not satisfy a pure recurrence: $E(7,11), E(8,10), E(8,12), E(10,15)$.

D. G. Cantor [6] has studied families of $E$-sequences of the type $E\left(a_{0}, a_{1}+a_{0}^{2} m\right)$ for integer $m$. He gives conditions under which each member of the family, for sufficiently large $m$, satisfies a recurrence. The family $E(7,49 m+15)$ is interesting since it contains the nonrecurrent $E(7,15)$ but it appears from [9] that $E(7,49 m+15)$ is recurrent for $m \geqslant 1$, with generating function $A(z) /(Q(z)-m z A(z))$, where $A(z)=7+$ $z+2 z^{2}-3 z^{3}$, and $Q(z)=1-2 z-z^{3}+z^{4}$. The polynomial $z^{4} Q\left(z^{-1}\right)$ is indeed the minimal polynomial for a $P$. V. number; but

$$
A(z) / Q(z)=7153268144 \ldots,
$$

while

$$
E(7,15)=7153268145 \ldots
$$

Observing that $(68)^{2} / 32=144 \frac{1}{2}$, we see that the "reason" $E(7,15)$ is a nontypical member of its family is that we have rounded $1441 / 2$ up to 145 rather than down to 144.

8. Finite Families of $S$ and $T$ Numbers. An interesting phenomenon was encountered at a number of points in our investigation, and is perhaps best illustrated by the following example. In an attempt to prove the nonrecurrence of $E(6,16)$, we considered a small interval containing $\theta(6,16)=2.69920$ 99139. An incomplete search of this interval revealed a number of polynomials of the form $\left(z^{n} P(z)+A(z)\right) /\left(z^{7}-1\right)$, where $P(z)=z^{5}-3 z^{4}+z^{3}-z-1$ and $A(z)=z^{8}+z^{5}+z^{4}-z^{2}+2 z-1$. If we let $B(z)=\left(z^{7}-1\right)(z-1)$, then a straightforward calculation shows that

$$
|P(z)|^{2}=|A(z)|^{2}+|B(z)|^{2} \quad \text { on }|z|=1 ;
$$


and hence, if $Q(z)=z^{5} P\left(z^{-1}\right)$, that $f(z)=A(z) / Q(z)$ is bounded by 1 on $|z|=1$. However, $P \notin S$ since, in addition to a root $\theta=2.6992099139$, it has roots $\alpha, \bar{\alpha}$ with $\alpha \simeq 1.007144 \exp i\left(51.56064^{\circ}\right)$.

By methods similar to the proof of Lemma 3.1 of [4], it can be shown that if $\omega$ is a root of $z^{n} P+A=0$ with $|\omega|=1$ and

$$
n<\operatorname{Re} \omega\left\{A^{\prime}(\omega) A(\omega)^{-1}-P^{\prime}(\omega) P(\omega)^{-1}\right\}=M(\omega),
$$

then $z^{n} P+A$ has exactly one root in $|z|>1$ and that this is then a member of $S$. From (43), the only choice for $\omega$ is either $\omega=1$, or else a 7th root of unity, and indeed, if $\omega=\exp (2 \pi i / 7)$, then $\omega^{n} P(\omega)+A(\omega)=0$ if $n \equiv 4(\bmod 7)$. Also, $M(\omega)=$ 111.7561 so that $\left(z^{n} P(z)+A(z)\right) /\left(z^{7}-1\right)$ is in $S$ if $n=7 k+4$ with $0 \leqslant k \leqslant 15$. We obtain a similar family by using $z^{8} A\left(z^{-1}\right)$ in place of $A(z)$.

The polynomial $P$ appeared in [9] as well. Galyean found that $E(6,16)$ was predicted for 45 terms by $\left(6-2 z+z^{2}+3 z^{3}+2 z^{4}\right) / z^{5} P\left(z^{-1}\right)$, but that it satisfies no recurrence of degree $\leqslant 361$ [9, pp. 18, 78].

By Rouche's theorem, all the polynomials $z^{n} P(z) \pm A(z)$ or $z^{n} P(z) \pm z^{8} A\left(z^{-1}\right)$ have at most three zeros in $|z|>1$ so these provide suitable polynomials for the method of Section 7 of [4]. For example,

$$
P_{0}(z)=z^{6} P(z)-z^{8} A\left(z^{-1}\right)=1-3111-3000-1-1000-1
$$

has roots $\theta=2.6791355714$ and $\alpha, \bar{\alpha}$ with $|\alpha|=1.000453$. Thus, $1+2(|\alpha|-1)^{-1}=$ 4418.46 so [4, Iemma 7.1] it is possible that $z^{m} P_{0}(z)+\epsilon z^{1}{ }^{1} P_{0}\left(z^{-1}\right), \epsilon= \pm 1$, produces Salem numbers for $m$ as large as 4418 . Indeed, with $\epsilon=-1$, Salem numbers are produced for all but 7 values of $m$ in $2 \leqslant m \leqslant 100$ and for $m=4184$; with $\epsilon=1$, Salem numbers are produced for all but 14 values of $m$ in $1 \leqslant m \leqslant 100$, and also for $m=4013,4020,4027,4034$.

Department of Mathematics

The University of British Columbia

Vancouver, British Columbia, Canada V6T 1W5

1. M. AMARA, “Ensembles fermés de nombres algébriques," Ann. Sci. École Norm. Sup. (3), v. 83, 1966, pp. 215-270.

2. P. E. BLANKSBY \& H. L. MONTGOMERY, "Algebraic integers near the unit circle," Acta Arith., v. 18, 1971, pp. 355-369.

3. D. W. BOYD, "Pisot sequences which satisfy no linear recurrence," Acta Arith., v. 32, 1977, pp. 89-98.

4. D. W. BOYD, “Small Salem numbers," Duke Math. J., v. 44, 1977, pp. 31 5-328.

5. D. W. BOYD, "Pisot numbers and the width of meromorphic functions." (Privately circulated manuscript.)

6. D. G. CANTOR, “On families of Pisot E-sequences," Ann. Sci. Ecole Norm. Sup. (4), v. 9, 1976, pp. 283-308.

7. J. DUFRESNOY \& Ch. PISOT, "Étude de certaines fonctions méromorphes bornées sur le cercle unité, application à un ensemble fermé d'entiers algébriques," Ann. Sci. Ecole Norm. Sup. (3), v. 72, 1955, pp. 69-92.

8. J. DUFRESNOY \& Ch. PISOT, "Sur les elements d'accumulation d'un ensemble fermé d'entiers algébriques," Bull. Sci. Math. (2), v. 79, 1955, pp. 54-64.

9. P. H. GALYEAN, On linear recurrence relations for E-sequences, Thesis, University of California, Los Angeles, 1971.

10. M. GRANDET-HUGOT, "Ensembles fermés d'entiers algébriques," Ann. Sci. École Norm. Sup. (3), v. 82, 1965 , pp. 1-35. 
11. D. E. KNUTH, The Art of Computer Programming. I, Addison-Wesley, Reading, Mass., 1968.

12. R. SALEM, "A remarkable class of algebraic integers. Proof of a conjecture of Vijayaraghavan," Duke Math. J., v. 11, 1944, pp. 103-107.

13. R. SALEM, "Power series with integral coefficients," Duke Math. J., v. 12, 1945, pp. $153-171$.

14. C. L. SIEGEL, "Algebraic integers whose conjugates lie in the unit circle," Duke Math. J., v. 11, 1944, pp. 597-602.

15. C. J. SMYTH, personal communication, March 10, 1977. 